\title{
Pathogenesis of endometriosis: The role of initial infection and subsequent sterile inflammation (Review)
}

\author{
HIROSHI KOBAYASHI, YUMI HIGASHIURA, HIROSHI SHIGETOMI and HIROTAKA KAJIHARA \\ Department of Obstetrics and Gynecology, Nara Medical University, Kashihara, Nara 634-8522, Japan
}

Received April 5, 2013; Accepted October 14, 2013

DOI: $10.3892 / \mathrm{mmr} .2013 .1755$

\begin{abstract}
Endometriosis is a common type of chronic inflammatory disease with an immunological background. In this review, we aimed to explore the contemporary literature on the infection and sterile inflammation that support the pathogenesis of endometriosis. This article reviews the English-language literature on inflammatory, environmental, immunological and oxidative factors associated with endometriosis in an effort to identify factors that cause a predisposition to endometriosis. Intrauterine microbes may be critical for the initiation of endometriosis; the initial activation of pathogen recognition receptors by microbial stimuli results in the activation of proinflammatory pathways and innate immunity. In addition to their response to various exogenous pathogen-associated molecular patterns, Toll-like receptors (TLRs) also recognize a wide range of endogenous danger-associated molecular patterns (DAMPs). The increased expression levels of DAMPs may be involved in the subsequent process of nuclear transcription factor- $\kappa \mathrm{B}$-dependent sterile inflammation. Oxidative stress, secondary to the influx of iron during retrograde menstruation, is involved in the progression of endometriosis. DAMP-mediated danger signals and oxidative stress are bidirectional during sterile inflammation (danger signal spiral). This review supports the hypothesis that there are at least two distinct phases of endometriosis development: The initial wave of TLR activation in modulating innate immune responses would be followed by the second big wave of sterile inflammation.
\end{abstract}

\section{Contents \\ 1. Introduction \\ 2. Study methodology}

Correspondence to: Professor Hiroshi Kobayashi, Department of Obstetrics and Gynecology, Nara Medical University, 840 Shijo-cho, Kashihara, Nara 634-8522, Japan

E-mail: hirokoba@naramed-u.ac.jp

Key words: cytokine, endometriosis, sterile inflammation, oxidative stress, iron, danger signal
3. Innate immunity and inflammation in endometriosis

4. Role of initial infection

5. Role of subsequent sterile inflammation

6. Oxidative stress

7. DAMPs

8. Antiapoptosis

9. Cytokines

10. Inflammasome

11. Novel treatment strategies

12. Antiapoptosis as a target

13. Inhibitors of DAMPs

14. Iron chelators

15. Conclusion

\section{Introduction}

Endometriosis is characterized by the growth of endometrial tissue outside the uterus. Endometriosis may result from increased cellular proliferation or a reduction in apoptosis (1). The balance between proliferation and apoptosis is changed in eutopic endometrium from endometriosis. Proposed hypothetical causes of endometriosis include retrograde menstruation, coelomic metaplasia and embryonic rest. Sampson's theory of retrograde menstruation hypothesizes that the mechanical transfer, invasive implantation and ectopic growth of endometrial tissues causes endometriosis and it remains the most widely accepted causal theory. However, no single theory is able to completely explain the origin and all aspects of this disorder (2). The mechanisms responsible for the initial development and subsequent progression of endometriosis are not clear.

Estrogen is involved in the development and progression of endometriosis. Treatment for endometriosis is expectant, medical or surgical. Medical treatment strategies focus on the creation of states of pseudopregnancy or pseudomenopause. However, the most crucial problem is that the current medical and surgical treatments of endometriosis are associated with high rates of relapse. Thus, a novel approach would be a beneficial improvement for the treatment of endometriosis. The identification of the pathogenesis of this condition is a crucial step for developing novel strategies for the treatment.

The present study reviews the contemporary literature on the infection and sterile inflammation that support the pathogenesis of endometriosis. 


\section{Study methodology}

The present study reviews the literature for biological studies on the development of endometriosis. Data pertaining to in vitro and in vivo studies were included. A computerized literature search was performed to identify relevant studies reported in the English language. All abstracts obtained from Medline electronic database were reviewed by two investigators (Y.H. and H.S.) to identify studies for full-text review. The web-based databases were searched by combining the keywords 'TLR', 'PAMP', 'inflammation' 'iron' 'oxidative stress' 'damage' 'DAMP' 'danger signal' 'NF- $\kappa \mathrm{B}$ ' or 'antiapoptosis' with 'endometriosis'. Additionally, references in each report were searched to identify potentially missed studies. Target publications were mainly studies on humans and animal models, as well as basic studies in gene and protein expression systems. Abstracts were not included, as they do not undergo a stringent peer review process.

\section{Innate immunity and inflammation in endometriosis}

Endometriosis is associated with angiogenesis, lymphangiogenesis and neurogenesis, which may be induced through inflammatory cell activation and contribute to the ectopic growth of endometrial tissue. Mounting evidence suggests that endometriosis is a common type of chronic inflammatory disease with an immunological background (3). Substantial numbers of immune cells, such as macrophages, neutrophils, dendritic cells, natural killer cells and mast cells, have been identified in peritoneal fluids associated with endometriosis (4) and macrophage activity may be fundamental in this disorder. However, these immune-associated cells fail to detect and eliminate ectopic endometrial cells, suggesting that they are dysfunctional (4).

The conditions for the development of endometriosis are induction of angiogenesis and lymphangiogenesis, which comprise a continuum of vascular development. Endometriotic lesions, particularly deep infiltrating endometriosis, have lymphangiogenic properties. Furthermore, mast cells are key mediators of allergic reactions of the immune system (5) and their immune function may extend far beyond this role (6). Endometriosis is a common cause of pelvic pain, possibly by regulating the recruitment of their own unique neural supplies through neurogenesis (7). Although the precise reason for the endometriosis-associated increase in neurogenesis is unknown, there is evidence of a closer proximity between mast cells and nerve fibers, demonstrating that mast cells are able to contribute to the development of pain $(5,8)$.

There is increasing evidence to demonstrate marked elevation of proinflammatory cytokines [interleukin (IL)-1 $\beta$ and tumor necrosis factor (TNF)- $\alpha$ ], angiogenic cytokines (leptin and IL-8), angiogenic growth factors (vascular endothelial growth factor and protein kinase CK2) and prostaglandin concentrations in endometriosis $(3,7,9)$. IL- $1 \beta$ and TNF- $\alpha$ in peritoneal fluid are able to activate c-Jun $\mathrm{N}$-terminal kinase (JNK) in eutopic endometrial cells from women with endometriosis, which in turn may upregulate inflammatory cytokine expression (10). JNK is also activated in response to cellular stress (10).
Endometriosis is often accompanied by marked changes in the number and function of inflammatory products, including human neutrophil peptides belonging to the $\alpha$-defensin family (11), macrophage migration inhibitory factor (MIF) (12), C-C chemokine monocyte chemoattractant protein-1 (MCP-1), serum amyloid A (SAA), TNF- $\alpha$, IL-1, IL-6, IL-8, chemokine (C-C motif) receptor 1 (CCR1) (13) and regulated on activation, normal $\mathrm{T}$ cell expressed and secreted (RANTES) (14). Defensin is involved in innate immunity against bacteria and kills microbes. MIF is a potent proinflammatory and growth-promoting factor and acts on ectopic endometrial cells to stimulate the production of COX-2 and $\mathrm{PGE}_{2}$. MCP-1 activates monocytes and recruits into the inflammation site. The hepatic biosynthesis of SAA is upregulated by proinflammatory cytokines. CCR1 is a CC chemokine receptor with high affinity for RANTES. TNF- $\alpha$ induces the expression of RANTES, which in turn, stimulates recruitment of macrophages into the endometriotic tissues. Angiogenic proinflammatory cytokines, leptin and IL-8 are potentially involved in the pathophysiology of endometriosis (3). Leptin produced by the adipose tissue regulates innate and adaptive immune responses and inflammation. IL-8 has been identified as a chemotactic factor for leukocytes and is also produced following inflammation. The levels of leptin and IL-8 are increased in endometriosis, reflecting inflammation and dysregulated immunomodulation (3). Angiopoietins, ligands of the endothelial TEK (Tie2) tyrosine kinase receptor, have been associated with angiogenesis (9). The Tie2-expressing macrophages regulate angiogenesis and lymphangiogenesis to maintain the viability of newly-formed vessels in endometriosis (9).

These data support the involvement of a chronic inflammatory state in endometriotic cells growing in the extra-uterine environment. Cytokines and growth factors may be significant in endometriosis-associated inflammation $(3,10,15)$. Principal molecular factors of inflammation-associated angiogenesis, lymphangiogenesis and neurogenesis should be identified to develop novel therapeutic strategies for this disorder.

\section{Role of initial infection}

Application of advances in genomic and proteomic technologies has provided molecular insights into endometriosis. Estrogen stimulates proliferation of endometrial and endometriotic cells. In addition to estrogen, the proliferation of an endometriotic lesion is regulated by the innate immune system. Innate immunity is used as a first defense against pathogens. A microbial infection of the upper genital tract may be critical for the initiation of chronic pelvic inflammation. Although the immune system is able to reject harmful pathogens, commensal microbes have coexisted with cells at the cell surfaces in a symbiosis (16). Disturbances in the maintenance of endometrial homeostasis and regulation of the host defense against bacterial infection lead to a break in the endometrial barrier function in genetically-susceptible hosts. Spontaneous contamination of Escherichia coli in menstrual blood and peritoneal fluid may promote Toll-like receptor (TLR) 4-mediated growth of endometrial tissue originating from retrograde menstruation (17). The TLR system responds immediately to infectious agents. The proinflammatory innate immune response leads to the activation of the slower 
adaptive immune system. It has also been reported that the TLR4 A896G polymorphism (rs4986790) is a functional polymorphism resulting in peritoneal inflammation (18). The initial development of endometriosis, e.g., adhesion and growth of ectopic endometrial tissue, may be characteristically non-sterile (Fig. 1, Step 1).

Host pathogen recognition receptors (PRRs) recognize microbial structures referred to as pathogen-associated molecular patterns (PAMPs) (16). TLRs are the first class of cell PRRs $(16,19)$. These PAMPs include lipopolysaccharide (LPS) and other components such as flagellin, a bioactive TLR5 ligand (16). PAMPs trigger intracellular signaling events, including ion influxes, cytosolic $\mathrm{Ca}^{2+}$ accumulation, production of reactive oxygen species (ROS) and phosphorylation of specific proteins, and their signaling pathways are involved in innate immunity through activation of TLRs. TLR activation induces key inflammatory mechanisms such as nuclear transcription factor $-\kappa \mathrm{B}(\mathrm{NF}-\kappa \mathrm{B})$ activation and the synthesis of IL-1 $\beta$ mRNA (19). A complex network of pathways is targeted by immunosuppressive cytokines such as IL-10 and transforming growth factor (TGF)- $\beta$.

TLR-mediated inflammation possibly occurs through the exogenous PAMPs as well as the endogenous danger-associated molecular pattern (DAMP) ligands (2). Nucleotide oligomerization domain-like receptors (NLRs) and, to a lesser degree, the retinoic acid-inducible gene I-like receptors are intracellular sensors (16). Activation of PRR signaling via members of the TLR and NLRs families initiates inflammatory defense mechanisms that are required to protect the host. NLRs respond to a variety of pathogen and intracellular danger signals (20) and induce the transcription of proinflammatory cytokines via the activation of NF- $\mathrm{B}$ (19). NLRC5, expressed through the signal transducers and activators of the transcription 1-mediated signaling pathway, has been identified as a regulator of NF- $\mathrm{NB}$, type I interferon (IFN) and inflammasome signaling pathways (see section 10) (20). NLRs also trigger mitogen-activated protein kinase signaling pathways and control the activation of inflammatory caspases and subsequent activation of proinflammatory cytokines (16). Activation of caspase- 1 is essential for the processing of pro-IL-1 $\beta$ and pro-IL-18 (16). In the absence of the anti-inflammatory feedback signals, physiological defense mechanisms may turn into pathological responses.

Gene expression profiling studies may reveal factors that explain variability in the development and progression of endometriosis. Notably, TLR activation in eutopic endometrium may be involved in the initial inflammatory response. The increased expression levels of several factors, including inflammatory cytokines and innate immunity, may be involved in the process of TLR-dependent inflammation (2). Khan et al studied the role of bacterial LPS and TLR4 in endometriosis, suggesting the association between bacterial infection and endometriotic proliferation (17). Bacterial LPS may have an initial role in the development of endometriosis.

\section{Role of subsequent sterile inflammation}

Inflammation is part of the non-specific immune response. In certain cases, the inflammatory process becomes continuous and the early endometriotic lesion may develop subsequently.
At least two waves of inflammation-induced gene expression occurs in endometriotic tissues. The first wave includes conserved infection and immune-associated early genes. The second-wave genes encode oxidative stress and sterile inflammatory factors required for proper regeneration. Therefore, the initial wave of the LPS-dependent TLR activation in modulating immune responses would be followed by a second wave of the mechanisms responsible for enhancing the oxidative stress and sterile inflammation.

\section{Oxidative stress}

Although the mechanism by which oxidative stress induces inflammation remains unclear, one prominent and early mediator for inflammation in endometriosis may be free iron (21). Redox active metals such as iron possess the ability to produce reactive radicals, for example the superoxide anion radical. Iron-induced inflammation is mediated through ROS production by driving the Fenton reaction (Fig. 1, Step 2). Excessive production of ROS is secondary to peritoneal influx of pro-oxidants such as iron during retrograde menstruation (22). Iron is able to induce oxidative stress in endometriosis during the secondary persistent inflammatory response (23). ROS induce cellular and DNA damage and increased proinflammatory gene expression through NF- $\kappa \mathrm{B}$ activation (22). In mice deficient for the NF- $\kappa \mathrm{B}$ family, endometriosis development was reduced (24). Iron also induces IL-1 $\beta$ expression, and the inhibition of IL-1 $\beta$ prevents development of endometriosis. These data allow us to hypothesize that the iron overload affects numerous mechanisms involved in the development of endometriosis during sterile inflammation. Iron and $\mathrm{NF}-\kappa \mathrm{B}$ appear to be connected, making these signaling pathways an attractive target for the future treatment of this disease (23). Sterile inflammation and oxidative stress by redox-active iron may lead to a circulus vitiosus, resulting in the persistence of inflammatory processes and the development of endometriosis when the process becomes chronic (2).

\section{DAMPs}

TLRs are a critical environmental interface that regulate infection and sterile injury by responding to a variety of microbial and endogenous ligands (25). In addition to their response to various exogenous PAMPs, TLRs recognize a wide range of endogenous DAMPs, including high-mobility group box protein 1 (HMGB1), heat shock protein 70 (HSP70), adenosine-5'-triphosphate (ATP), DNA, urate crystals, asbestos, silica, aluminum hydroxide, S100, neutrophil elastase, amyloid- $\beta$ and soluble extracellular matrix components, such as biglycan, hyaluronan, versican, fibrinogen, heparan sulfate fragments and fibronectin extra domain A $(2,26,27)$. Intracellular contents released from damaged cells into the extracellular space serve as DAMPs or alarmins that trigger inflammation (Fig. 1, Step 3). Infection and damage cause inflammation as PAMPs as well as DAMPs are immunogenic. There is increasing evidence that this sterile inflammatory response mediated through DAMPs is a key determinant of further development of inflammation-associated diseases such as atherosclerosis, gout, type II diabetes and pancreatitis (26). A number of DAMPS, including HMGB1, DNA, 
ATP and HSP70, have been shown to be involved in endometriosis (2). The TLR-mediated inflammation persistently occurs possibly through the endogenous ligands. Persistent sterile inflammatory insults and DAMPs released from damaged cells are bidirectional, and they activate immunity and further propagate tissue damage. Specific DAMP receptors, including TLR4, TLR9 and P2X7, as well as downstream DAMP-sensing components, including NLRP3, caspase-1, IL-1 $\beta$, IL-18 and IL-1 receptor, are required for full experimental endometriosis (26). These DAMP-mediated pathways may provide novel therapeutic targets.

Mesothelial cells undergo injury and repair themselves following retrograde menstruation-associated inflammation. Peritoneal endometriosis is the result of ectopic implantation and growth of endometrial tissue in women with a deficient immune system, which is not able to defend against regurgitated endometrial cells (28). Specific factors, including TNF- $\alpha, \alpha$-enolase and hemoglobin, in menstrual effluent induce epithelial to mesenchymal transitions (EMT) in mesothelial cells, resulting in cell retraction and exposure of the submesothelial extracellular matrix (ECM) (28). Structural damage of the mesothelial layer attributable to the menstrual effluent may facilitate regurgitated endometriotic cell adhesion and growth. Fibrosis around the endometriotic foci is the consequence of recurrent cell damage. Endometriotic cell migration and the concomitant degradation of ECM are two essential steps in the invasive process. ECM components such as biglycan, hyaluronan, versican, fibrinogen, heparan sulfate fragments and fibronectin extra domain A have now been demonstrated to act as signaling molecules (29). They act as fundamental danger signals or DAMPs, signifying tissue injury, and they also potentiate the immune system (29). The expression of certain danger signal proteins involved in the organization of the cytoskeleton, signal transduction, regulation of the redox state and production of ATP, has been demonstrated to be altered during the EMT process.

ATP is a ubiquitous molecule in every cell and is released into the extracellular milieu following tissue injury. Extracellular ATP is a host-derived small-danger-molecule. ATP synthase $\beta$ subunit has been demonstrated to be differentially expressed between women with and without endometriosis. ATP synthase $\beta$-chain has been identified as phosphorylated and activated in endometriosis. Thus, extracellular ATP released from ectopic endometrial cells may constitute a major endogenous danger signal that leads to IL-1 $\beta$ expression and fibrosis.

Although DAMPs such as endogenous DNA and nuclear HMGB1 have been shown to be critical in sterile inflammation, the role of nuclear histone proteins has not yet been investigated in endometriosis. Numerous studies (26) have established that certain non-histone proteins such as HMGB1 are released extracellularly and induce innate/inflammatory and adaptive immune responses as a DAMP. DNA binds histones to form nucleosomes. Ischemia/reperfusion (I/R) injury of several organs enhances histone expression and selected proinflammatory/profibrotic genes. Injection of exogenous histones exacerbates injury through the TLR9 and MyD88-mediated cytotoxic effects, and histone neutralization protects against injury (30). Histones function as a DAMP following I/R injury (30). We hypothesize that histones may be a novel class of DAMP molecules and serve as an activator of innate immunity during sterile inflammation in endome- triosis. These findings require additional preclinical studies on DAMPs in endometriosis.

\section{Antiapoptosis}

The release of DAMPs from endometriotic-damaged cells may engender a second wave of tissue damage during acute processes and, if chronic, potentially trigger antiapoptotic processes. Increased levels of cell proliferation and reduced levels of apoptosis emerge as major mechanisms responsible for the development of endometriosis. Attenuated susceptibility to apoptosis may contribute to the pathogenesis of endometriosis $(31,32)$. Marked cell proliferation has been attributed to a change in the expression levels of proteins such as B-cell lymphoma-2 (Bcl-2) and Bcl-2-associated X proteins (Bcl-XL, Bcl-XS). A number of other genes are associated with apoptosis in endometriosis, including defender against cell death-1, P53, survivin, caspase-1, calpain, proliferating cell nuclear antigen and $\mathrm{PGE}_{2}$ synthesis genes (31,33-35). $\mathrm{PGE}_{2}$ promotes cell survival through the EP2/EP4 receptor-dependent activation of ERK1/2, Akt, NF- $\mathrm{BB}$, and $\beta$-catenin signaling pathways, suppressing proapoptotic proteins (Bax and Bad) and enhancing antiapoptotic proteins (Bcl-2/Bcl-XL) (33). The $\mathrm{PGE}_{2}$ signaling components are abundantly expressed in endometriosis (33). COX-2 induces $\mathrm{PGE}_{2}$ expression through the cAMP/ERK pathways by activating EP2 and EP4 receptors. COX-2 also reduces apoptosis-associated caspase-3 expression (36). Selective COX-2 inhibition induces regression of endometrial grafts by stimulation of apoptosis (36). Decreased apoptosis is also associated with a pathway involving HOXA10, calpain5 and caspase (37). Expression levels of calpain5, a target of HOXA10 transcriptional regulation, are reduced in endometriosis which is likely to be the result of reduced HOXA10 expression levels (37).

\section{Cytokines}

Commonly upregulated cytokines in endometriosis include IL- $1 \beta$, TNF- $\alpha$, IFN- $\gamma$, IL-18, IL-10 and TGF- $\beta$. IL- $1 \beta$ and IL-18 are two powerful proinflammatory cytokines with pleiotropic activities. IL-18 increases natural killer cell activity, and stimulates IFN- $\gamma$ production in T-helper type I cells. IL-18 is involved in various immune diseases and induces COX-2 in peritoneal monocytes (38). Although there are a number of studies on the association between IL-18 and endometriosis, their results appear to be contradictory (38).

Upregulation of IL-10 expression levels was also observed in ovarian endometrioma when compared with a control group (39). The biological actions of IL-10 are inhibitory, including the inactivation of macrophages and inhibition of proinflammatory cytokines, demonstrating that IL-10 functions as an immunoregulatory cytokine.

Host-derived TGF- $\beta 1$ is a multifunctional cytokine that is upregulated in endometriosis (40). TGF- $\beta 1$ induces the secretion of IL-6 from endometrial stromal cells through an increase in protease-activated receptor (PAR)-2 expression levels (41). TGF- $\beta 1$ may enhance the development of endometriosis (40).

$\mathrm{NF}-\kappa \mathrm{B}$ is involved in the transduction of proinflammatory signals, upregulation of adhesion molecules and suppression of apoptosis, thus enhancing the initial development of endome- 


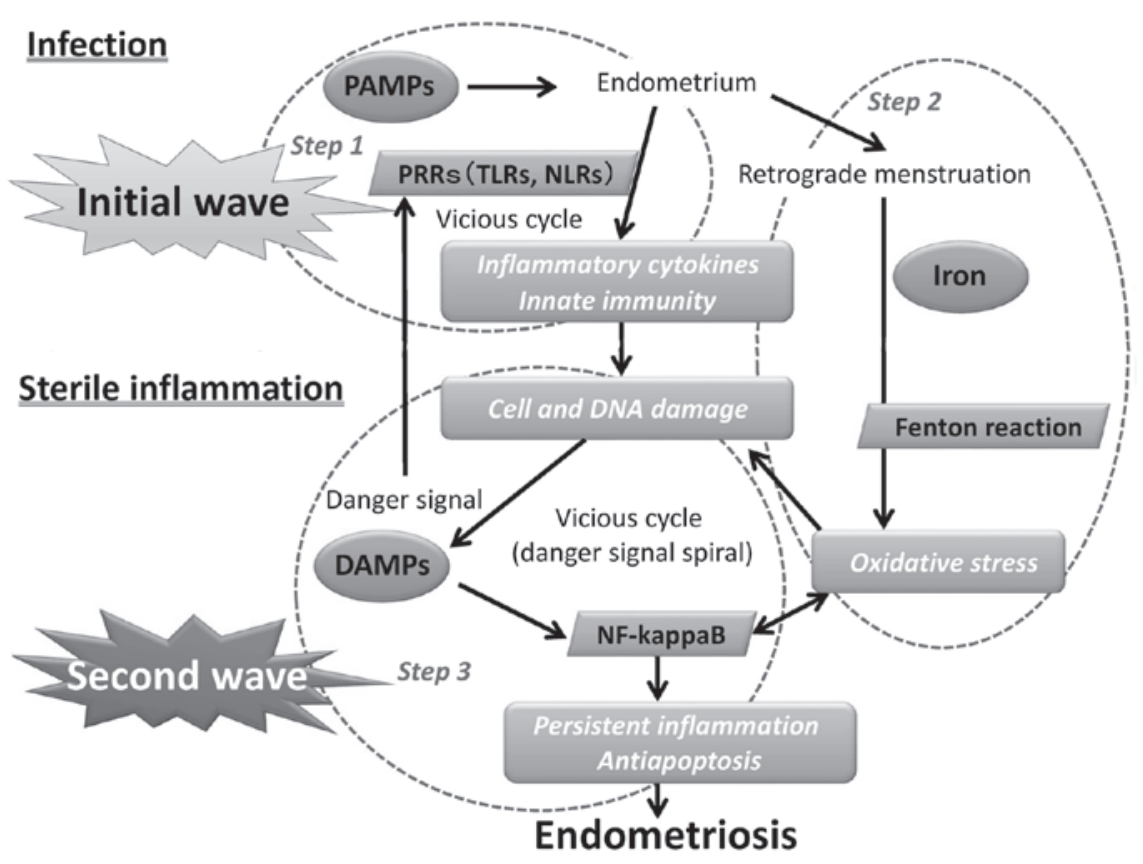

Figure 1. Initial infection and subsequent sterile inflammation are essential for the development and progression of endometriosis. (Step 1) Spontaneous contamination of Escherichia coli in menstrual blood may promote TLR-mediated growth of endometrial tissue originating from retrograde menstruation. TLRs recognize microbial structures referred to as PAMPs. TLR activation is involved in the initial inflammatory response and innate immunity. (Step 2) Excessive production of ROS through the Fenton reaction is secondary to the peritoneal influx of free iron during retrograde menstruation. (Step 3) ROS induce cell and DNA damage and increased proinflammatory gene expression through the DAMP-mediated NF- $\kappa B$ activation. Therefore, the initial wave of the LPS-dependent TLR activation in modulating immune responses would be followed by a second wave of the mechanisms responsible for enhancing the oxidative stress and sterile inflammation. TLR, toll-like receptor; PAMPs, pathogen-associated molecular patterns; ROS, reactive oxygen

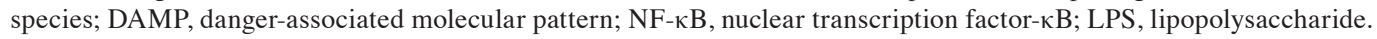

triosis (43). NF- $\kappa \mathrm{B}$, as a series of cascade events of an autocrine nature, regulates the expression of proinflammatory cytokines and activation of $\mathrm{NF}-\kappa \mathrm{B}$ itself, leading to the maintenance of autocrine self-amplifying cycles (Fig. 1).

\section{Inflammasome}

Production of pro-IL-1 $\beta$, an inactive precursor, is induced by the activation of $\mathrm{NF}-\kappa \mathrm{B}$ in response to inflammatory stimuli. In the generation of IL-1 $\beta$, additional proteolytic cleavage by cytosolic protein complexes termed 'inflammasomes' is required for the activation of pro-IL-1 $\beta$ (16). Inflammasomes assemble in response to endogenous danger signals. Caspase- 1 is auto-activated within inflammasomes that include NLR proteins, the adapter apoptosis-associated speck-like protein containing a C-terminal caspase recruitment domain and pro-caspase-1 (16). Accumulating evidence indicates that several sterile inflammatory responses triggered by oxidative stress or cell damage are mediated by inflammasomes. Subsequent activation of inflammasomes leads to $\mathrm{NF}-\kappa \mathrm{B}$ activation and IL-1 $\beta$ production, resulting in inflammatory responses. Therefore, we hypothesize that the inflammasome is an initial sensor for danger signals in endometriosis. However, regulation of the inflammasome formation in endometriosis is poorly understood.

\section{Novel treatment strategies}

This review allows us to hypothesize that several promising therapies that target DAMPs in antiapoptosis, sterile inflammation and oxidative stress should be developed. Novel treatments from genomic investigations may be identified due to the accumulation of a therapeutic signature for endometriosis. A high-throughput screening assay is capable of revealing a number of compounds that specifically inhibit a target molecule activity.

\section{Antiapoptosis as a target}

Antiapoptosis may be involved in the pathophysiology of endometriosis (32). Accordingly, apoptosis of the nascent endometriotic lesion has become an attractive target to use to inhibit the development of endometriosis. An array of promising therapeutic agents, including BAY 11-7085, $\beta$-hydroxyisovalerylshikonin ( $\beta$-HIVS), bufalin and rapamycin, have entered various stages of preclinical development for endometriosis. Bay 11-7085 is reported to inhibit $\mathrm{NF}-\kappa \mathrm{B}$ activation and this pharmacological inhibitor induces apoptosis through the suppression of antiapoptotic proteins such as caspase-3, -8 , and -9 (43). Extract of the roots of Onosma paniculata, $\beta$-HIVS, induces apoptosis in cancer cells (44). $\beta$-HIVS is an ATP non-competitive inhibitor of protein-tyrosine kinases. It induces G0/G1 phase cell-cycle arrest and apoptosis via the downregulation of Bcl-2 expression with the activation of caspase- $3,-8$ and -9 , leading to the inhibition of endometriotic cell proliferation (44). Bufalin, an apoptosis-inducing agent, is a major digoxin-like compound isolated from the skin and parotid venom glands of toads (35). Rapamycin is a drug with antifungal, immunosuppressant, antiapoptotic and antiangiogenic effects (45). The apoptotic 
activities of these agents may be sufficient for additional clinical investigation on the development of endometriosis.

\section{Inhibitors of DAMPs}

Molecules of DAMPs are stimulated under conditions of stress, such as injury, infection, ischemia or oxidative stress. They induce immune tolerance or various chronic diseases such as arthritis, atherosclerosis, cancer, systemic lupus erythematosus and possibly endometriosis. These data suggest a role for DAMP molecules in the enhanced and persistent activation of endometriosis. HMGB1 functions as a major DAMP and inhibits apoptosis, promoting Bcl-2 expression and inhibiting Bax translocation. Gabexate mesilate, a synthetic protease inhibitor with a degree of anti-inflammatory action, inhibits PAI-1 and PAR-2, thereby indirectly inhibiting HMGB1. HSP70 is a molecular chaperone that protects cells from damage in response to various stress stimuli (46). Preclinical studies suggest that the use of specific inhibitors for HSP70 is an effective strategy to treat Alzheimer's disease. Statin, a HMG-CoA reductase inhibitor, also inhibits the expression of HSP70 through the inactivation of NF- $\kappa$ B. The present findings indicate that DAMP molecules released from damaged endometriotic cells may serve as a link between the initial cell damage induced by bacterial infection and the subsequent activation of innate immune cells by sterile inflammation and oxidative stress. Direct or indirect inhibitors of DAMP molecules may be a potential therapeutic target to prevent or minimize endometriosis.

\section{Iron chelators}

Hemolysis occurring during the development of endometriosis results in high levels of free iron. Redox-active iron participates in the generation of highly toxic free radicals and oxidatively leads to cell and DNA damage. A previous study demonstrated the molecular basis for the initial inflammatory response following iron-induced oxidative stress and suggested that iron is a potential novel therapeutic target for preventing the development of endometriosis (21). Iron chelator treatment may be beneficial in endometriosis via the suppression of cell proliferation (47).

\section{Conclusion}

Based on epidemiological and experimental data, it is possible to a certain extent to hypothesize that retrograde menstruation promotes implantation and the development of endometrial tissue, in accordance with Sampson's hypothesis. The mechanism of the initial development and subsequent progression of endometriosis is largely unknown. There may be at least two waves of development of endometriosis; the first wave due to bacterial infection and the second wave from sterile inflammation (Fig. 1). The initial stage of a non-specific bacterial infection, such as E. coli, is believed to occur in the uterine endometrium and peritoneal fluid (Step 1). Oxidative stress is secondary to the influx of iron during retrograde menstruation (Step 2). Redox-active iron-dependent oxidative stress and PAMP/DAMP-receptor signaling (Step 3) provide the combination of antiapoptosis and persistent inflammation. The release of DAMPs from damaged cells may engender a second big wave of tissue damage during chronic processes and potentially trigger sterile inflammation-induced antiapoptotic and oxidative stress processes. In conclusion, initial infection and subsequent sterile inflammation are closely associated with the development of endometriosis.

\section{Acknowledgements}

This study was supported by a Grant-in-Aid for Scientific Research to H.K. from the Ministry of Education, Culture, Sports, Science and Technology (Tokyo, Japan).

\section{References}

1. Agic A, Djalali S, Diedrich K and Hornung D: Apoptosis in endometriosis. Gynecol Obstet Invest 68: 217-223, 2009.

2. Kajihara H, Yamada Y, Kanayama S, Furukawa N, Noguchi T, Haruta S, Yoshida S, Sado T, Oi H and Kobayashi H: New insights into the pathophysiology of endometriosis: from chronic inflammation to danger signal. Gynecol Endocrinol 27: 73-79, 2011.

3. Malhotra N, Karmakar D, Tripathi V, Luthra K and Kumar S: Correlation of angiogenic cytokines-leptin and IL-8 in stage, type and presentation of endometriosis. Gynecol Endocrinol 28: 224-227, 2012.

4. Khoufache K, Michaud N, Harir N, Kibangou Bondza P and Akoum A: Anomalies in the inflammatory response in endometriosis and possible consequences: a review. Minerva Endocrinol 37: 75-92, 2012.

5. Kirchhoff D, Kaulfuss S, Fuhrmann U, Maurer M and Zollner TM: Mast cells in endometriosis: guilty or innocent bystanders? Expert Opin Ther Targets 16: 237-241, 2012.

6. Menzies FM, Shepherd MC, Nibbs RJ and Nelson SM: The role of mast cells and their mediators in reproduction, pregnancy and labour. Hum Reprod Update 17: 383-396, 2011.

7. Asante A and Taylor RN: Endometriosis: the role of neuroangiogenesis. Annu Rev Physiol 73: 163-182, 2011.

8. D'Cruz OJ and Uckun FM: Targeting mast cells in endometriosis with janus kinase 3 inhibitor, JANEX-1. Am J Reprod Immunol 58: 75-97, 2007.

9. Capobianco A, Monno A, Cottone L, Venneri MA, Biziato D, Di Puppo F, Ferrari S, De Palma M, Manfredi AA and Rovere-Querini P: Proangiogenic Tie2(+) macrophages infiltrate human and murine endometriotic lesions and dictate their growth in a mouse model of the disease. Am J Pathol 179: 2651-2659, 2011.

10. Uz YH, Murk W, Bozkurt I, Kizilay G, Arici A and Kayisli UA: Increased c-Jun N-terminal kinase activation in human endometriotic endothelial cells. Histochem Cell Biol 135: 83-91, 2011.

11. Milewski Ł, Dziunycz P, Barcz E, Radomski D, Roszkowski PI, Korczak-Kowalska G, Kamiński P and Malejczyk J: Increased levels of human neutrophil peptides 1,2, and 3 in peritoneal fluid of patients with endometriosis: association with neutrophils, T cells and IL-8. J Reprod Immunol 91: 64-70, 2011.

12. Carli C, Metz CN, Al-Abed Y, Naccache PH and Akoum A: Up-regulation of cyclooxygenase-2 expression and prostaglandin E2 production in human endometriotic cells by macrophage migration inhibitory factor: involvement of novel kinase signaling pathways. Endocrinology 150: 3128-3137, 2009.

13. Agic A, Xu H, Finas D, Banz C, Diedrich K and Hornung D: Is endometriosis associated with systemic subclinical inflammation? Gynecol Obstet Invest 62: 139-147, 2006.

14. Lebovic DI, Chao VA and Taylor RN: Peritoneal macrophages induce RANTES (regulated on activation, normal T cell expressed and secreted) chemokine gene transcription in endometrial stromal cells. J Clin Endocrinol Metab 89: 1397-1401, 2004.

15. Michaud N, Al-Akoum M, Gagnon G, Girard K, Blanchet P, Rousseau JA and Akoum A: Decreased concentrations of soluble interleukin-1 receptor accessory protein levels in the peritoneal fluid of women with endometriosis. J Reprod Immunol 92: 68-73, 2011.

16. Franchi L, Warner N, Viani K and Nuñez G: Function of Nod-like receptors in microbial recognition and host defense. Immunol Rev 227: 106-128, 2009. 
17. Khan KN, Kitajima M, Hiraki K, Yamaguchi N, Katamine S, Matsuyama T, Nakashima M, Fujishita A, Ishimaru T and Masuzaki H: Escherichia coli contamination of menstrual blood and effect of bacterial endotoxin on endometriosis. Fertil Steril 94: 2860-2863, 2010.

18. Latha M, Vaidya S, Movva S, Chava S, Govindan S, Govatati S, Banoori M, Hasan Q and Kodati VL: Molecular pathogenesis of endometriosis; Toll-like receptor-4 A896G (D299G) polymorphism: a novel explanation. Genet Test Mol Biomarkers 15 181-184, 2011.

19. Brown J, Wang H, Hajishengallis GN and Martin M: TLR-signaling networks: an integration of adaptor molecules, kinases, and cross-talk. J Dent Res 90: 417-427, 2011.

20. Tong Y, Cui J, Li Q, Zou J, Wang HY and Wang RF: Enhanced TLR-induced NF- $\kappa \mathrm{B}$ signaling and type I interferon responses in NLRC5 deficient mice. Cell Res 22: 822-835, 2012.

21. Kobayashi H, Yamada Y, Kanayama S, Furukawa N, Noguchi T, Haruta S, Yoshida S, Sakata M, Sado T and Oi H: The role of iron in the pathogenesis of endometriosis. Gynecol Endocrinol 25: 39-52, 2009.

22. Lousse JC, Van Langendonckt A, Defrere S, Ramos RG, Colette S and Donnez J: Peritoneal endometriosis is an inflammatory disease. Front Biosci (Elite Ed) 4: 23-40, 2012.

23. Defrère S, González-Ramos R, Lousse JC, Colette S, Donnez O, Donnez $\mathbf{J}$ and Van Langendonckt $\mathrm{A}$ : Insights into iron and nuclear factor-kappa B (NF-kappaB) involvement in chronic inflammatory processes in peritoneal endometriosis. Histol Histopathol 26: 1083-1092, 2011.

24. Lu Y, Sun Q, Zheng Y, Liu X, Geng JG and Guo SW: The role of nuclear factor-kappa-B p50 subunit in the development of endometriosis. Front Biosci (Elite Ed): 591-603, 2011.

25. Mollen KP, Anand RJ, Tsung A, Prince JM, Levy RM and Billiar TR: Emerging paradigm: toll-like receptor 4-sentinel for the detection of tissue damage. Shock 26: 430-437, 2006

26. Hoque R, Malik AF, Gorelick F and Mehal WZ: Sterile inflammatory response in acute pancreatitis. Pancreas 41: 353-357, 2012.

27. Zeiser R, Penack O, Holler E and Idzko M: Danger signals activating innate immunity in graft-versus-host disease. J Mol Med (Berl) 89: 833-845, 2011

28. Demir AY, Demol H, Puype M, de Goeij AF, Dunselman GA, Herrler A, Evers JL, Vandekerckhove J and Groothuis PG: Proteome analysis of human mesothelial cells during epithelial to mesenchymal transitions induced by shed menstrual effluent. Proteomics 4: 2608-2623, 2004.

29. Schaefer L: Extracellular matrix molecules: endogenous danger signals as new drug targets in kidney diseases. Curr Opin Pharmacol 10: 185-190, 2010.

30. Huang H, Evankovich J, Yan W, Nace G, Zhang L, Ross M, Liao X, Billiar T, Xu J, Esmon CT and Tsung A: Endogenous histones function as alarmins in sterile inflammatory liver injury through Toll-like receptor 9 in mice. Hepatology 54: 999-1008, 2011

31. Watanabe A, Taniguchi F, Izawa M, Suou K, Uegaki T, Takai E, Terakawa $\mathrm{N}$ and Harada T: The role of survivin in the resistance of endometriotic stromal cells to drug-induced apoptosis. Hum Reprod 24: 3172-3179, 2009.

32. Izawa M, Harada T, Deura I, Taniguchi F, Iwabe T and Terakawa N: Drug-induced apoptosis was markedly attenuated in endometriotic stromal cells. Hum Reprod 21: 600-604, 2006.

33. Banu SK, Lee J, Speights VO Jr, Starzinski-Powitz A and Arosh JA: Selective inhibition of prostaglandin E2 receptors EP2 and EP4 induces apoptosis of human endometriotic cells through suppression of ERK1/2, AKT, NFkappaB, and beta-catenin pathways and activation of intrinsic apoptotic mechanisms. Mol Endocrinol 23: 1291-1305, 2009.
34. Braun DP, Ding J, Shaheen F, Willey JC, Rana N and Dmowski WP: Quantitative expression of apoptosis-regulating genes in endometrium from women with and without endometriosis. Fertil Steril 87: 263-268, 2007

35. Nasu K, Nishida M, Ueda T, Takai N, Bing S, Narahara $H$ and Miyakawa I: Bufalin induces apoptosis and the G0/G1 cell cycle arrest of endometriotic stromal cells: a promising agent for the treatment of endometriosis. Mol Hum Reprod 11: 817-823, 2005

36. Laschke MW, Elitzsch A, Scheuer C, Vollmar B and Menger MD: Selective cyclo-oxygenase-2 inhibition induces regression of autologous endometrial grafts by down-regulation of vascular endothelial growth factor-mediated angiogenesis and stimulation of caspase-3-dependent apoptosis. Fertil Steril 87: 163-171, 2007.

37. Penna I, Du H, Ferriani R and Taylor HS: Calpain5 expression is decreased in endometriosis and regulated by HOXA10 in human endometrial cells. Mol Hum Reprod 14: 613-618, 2008.

38. Oku H, Tsuji Y, Kashiwamura SI, Adachi S, Kubota A, Okamura $\mathrm{H}$ and Koyama K: Role of IL-18 in pathogenesis of endometriosis. Hum Reprod 19: 709-714, 2004.

39. Podgaec S, Dias Junior JA, Chapron C, Oliveira RM, Baracat EC and Abrão MS: Th1 and Th2 immune responses related to pelvic endometriosis. Rev Assoc Med Bras 56: 92-98, 2010.

40. Hull ML, Johan MZ, Hodge WL, Robertson SA and Ingman WV: Host-derived TGFB1 deficiency suppresses lesion development in a mouse model of endometriosis. Am J Pathol 180: 880-887, 2012.

41. Saito A, Osuga Y, Yoshino O, Takamura M, Hirata T, Hirota Y, Koga K, Harada M, Takemura Y, Yano T and Taketani Y: TGF- $\beta 1$ induces proteinase-activated receptor 2 (PAR2) expression in endometriotic stromal cells and stimulates PAR2 activation-induced secretion of IL-6. Hum Reprod 26: 1892-1898, 2011.

42. González-Ramos R, Van Langendonckt A, Defrère S, Lousse JC, Mettlen M, Guillet A and Donnez J: Agents blocking the nuclear factor-kappaB pathway are effective inhibitors of endometriosis in an in vivo experimental model. Gynecol Obstet Invest 65: 174-186, 2008.

43. Nasu K, Nishida M, Ueda T, Yuge A, Takai N and Narahara H: Application of the nuclear factor-kappaB inhibitor BAY 11-7085 for the treatment of endometriosis: an in vitro study. Am J Physiol Endocrinol Metab 293: E16-E23, 2007.

44. Nishida M, Nasu K, Ueda T, Yuge A, Takai N and Narahara H: Beta-hydroxyisovalerylshikonin induces apoptosis and G0/G1 cell-cycle arrest of endometriotic stromal cells: a preliminary in vitro study. Hum Reprod 21: 2850-2856, 2006.

45. Laschke MW, Elitzsch A, Scheuer C, Holstein JH, Vollmar B and Menger MD: Rapamycin induces regression of endometriotic lesions by inhibiting neovascularization and cell proliferation. Br J Pharmacol 149: 137-144, 2006.

46. Khan KN, Kitajima M, Imamura T, Hiraki K, Fujishita A, Sekine I, Ishimaru T and Masuzaki $\mathrm{H}$ : Toll-like receptor 4-mediated growth of endometriosis by human heat-shock protein 70. Hum Reprod 23: 2210-2219, 2008.

47. Defrère S, Van Langendonckt $A$, Vaesen S, Jouret $M$, González Ramos R, Gonzalez D and Donnez J: Iron overload enhances epithelial cell proliferation in endometriotic lesions induced in a murine model. Hum Reprod 21: 2810-2816, 2006. 